\title{
RETRACTED ARTICLE: Beta-cyanoalanine synthase pathway as a homeostatic mechanism for cyanide detoxification as well as growth and development in higher plants
}

\author{
Hafiz Mamoon Rehman' ${ }^{1}$ Zahid Hussain Shah ${ }^{2} \cdot$ \\ Muhammad Amjad Nawaz ${ }^{1}$ Muhammad Qadir Ahmad ${ }^{4}$. \\ Seung Hwan Yang ${ }^{1}$ Kang Hee $\mathrm{Kho}^{3} \cdot$ Gyuhwa Chung $^{1}$ (D)
}

Received: 27 July 2016/ Accepted: 9 October 2016/Published online: 15 October 2016

(c) Springer-Verlag Berlin Heidelberg 2016

This review article has been retracted at the request of the Editors-in-Chief and the Reviews Editor. It has been using the published $\mathrm{PhD}$ thesis "Activity of the beta-cyanoalanine synthase pathway is associated with the response to abiotic stress by Arabidopsis thaliana" by Marylou Machingura as template without making reference to it.
The original work can be consulted at http://opensiuc.lib. siu.edu/dissertations. The authors apologize to the Editors and readers as well as to the author of the original work. The online version of this article contains the full text of the retracted article as electronic supplementary material.

Electronic supplementary material The online version of this article (doi:10.1007/s00425-016-2606-3) contains supplementary material, which is available to authorized users.

\section{Gyuhwa Chung}

chung@chonnam.ac.kr

1 Department of Biotechnology, Chonnam National University, Yeosu, Chonnam 59626, South Korea

2 Department of Arid Land Agriculture, King Abdul-Aziz University, Jeddah 21577, Saudi Arabia

3 Department of Aquatic Biology, Chonnam National University, Yeosu, Chonnam 59626, South Korea

4 Department of Plant Breeding and Genetics, Bahauddin Zakariya University, Multan 6000, Pakistan 